\title{
Nonlinear Dynamics Analysis and Global Stabilization of Underactuated Horizontal Spring-Coupled Two-Link Manipulator
}

\author{
Ancai Zhang $\mathbb{D}^{1},{ }^{1}$ Jinhua She $\mathbb{D}^{2,3}{ }^{2,3}$ Zhenxing Li, ${ }^{1}$ Guochen Pang, ${ }^{1}$ Zhi Liu, ${ }^{1}$ \\ and Jianlong Qiu ${ }^{1,4}$ \\ ${ }^{1}$ School of Automation and Electrical Engineering, \\ Linyi University-Key Laboratory of Complex Systems and Intelligent Computing in Universities of Shandong, Linyi, \\ Shandong 276000, China \\ ${ }^{2}$ School of Engineering, Tokyo University of Technology, Hachioji, Tokyo 192-0982, Japan \\ ${ }^{3}$ School of Automation, China University of Geosciences, Wuhan, Hubei 430074, China \\ ${ }^{4}$ Department of Information Technology, King Abdulaziz University, Jeddah, Saudi Arabia \\ Correspondence should be addressed to Ancai Zhang; zhangancai123@hotmail.com
}

Received 25 August 2019; Revised 30 January 2020; Accepted 10 February 2020; Published 16 March 2020

Academic Editor: Alex Alexandridis

Copyright (c) 2020 Ancai Zhang et al. This is an open access article distributed under the Creative Commons Attribution License, which permits unrestricted use, distribution, and reproduction in any medium, provided the original work is properly cited.

\begin{abstract}
An underactuated horizontal spring-coupled two-link manipulator (UHSTM) is a nonlinear system that has one actuator and two degrees of freedom (DOF). This paper analyzes the nonlinear dynamics and develops a new global stabilization control method for this 2-DOF underactuated system. First, a set of suitable state variables are constructed to change the UHSTM system into a cascade nonlinear system. Second, a Lyapunov function is constructed based on the structural characteristics of the cascade system. A control law is designed to ensure the closed-loop control system to be Lyapunov stable. After that, the global asymptotical stability conditions are derived from the analysis of the closed-loop control system. Finally, numerical examples demonstrate the validity of the theoretical analysis results.
\end{abstract}

\section{Introduction}

With the improvement of industrial automation, the manipulators have been widely used in many areas of engineering [1-4]. For a manipulator, the damage of actuators is an inevitable problem caused by the wearing of devices and the influence of the surrounding environment. The failure of actuators results in that the manipulator becomes an underactuated mechanical system [5-7], in which the system has less number of actuators than degrees of freedom (DOF). Since an underactuated manipulator consumes less energy, it has a simple structure and is lightweight, and it also has good potential applications in many fields. It is meaningful to study the control of this kind of mechanical system [8-11]. However, this problem is challenging due to the complex inherent nonlinear dynamics and nonholonomic constraints possessed by this kind of system [12].

Over the past few years, a great deal of efforts have been made on the control of underactuated manipulators [13-15]. Among them, a two-link manipulator that has only one actuator is the simplest example. According to whether the manipulator is affected by gravity or not, the underactuated two-link manipulators are divided into two categories: underactuated vertical two-link manipulators (UVTM) and underactuated horizontal two-link manipulators (UHTM). The UVTM moves in a vertical plane [16], which includes Acrobot [17] and Pendubot [18]. Many control strategies have been presented to solve the stabilizing control of the UVTM, e.g., a partial feedback linearization method $[19,20]$, a fuzzy control method [21], an 
energy-based method [22, 23], and an equivalent-inputdisturbance (EID) method [24]. In contrast, the UHTM moves in a horizontal plane and is not affected by the gravity. It is clear that the UVTM becomes the UHTM in a weightless environment. Although the UHTM has the same mechanical configuration as the UVTM, they have quite different dynamic properties. The absence of gravity makes the UHTM system possess some unique properties. For example, any point in the motion space is an equilibrium point of the UHTM, and the system is not local controllable and even not small-time local controllable around the equilibrium point [25]. All these make the control of the UHTM be a very difficult problem.

In order to solve this control problem, some attempts have been made to find a gravity substitute for the UHTM. In [26], a spring was added around the passive joint of the UHTM, and an underactuated horizontal spring-coupled two-link manipulator (UHSTM) was constructed (see Figure 1). The UHSTM uses the spring to create a source of potential energy. It makes the control of the underactuated horizontal manipulator be easy to solve. In addition, the cost of a spring is much lower than an actuator, and the elasticity of the spring does not disappear in a weightless environment. Therefore, the UHSTM has a good application prospect in industrial manufacturing, exploration of outer space, and other fields.

Currently, there are few results about the control of the UHSTM system. In order to promote its application in the engineering areas, it is necessary to analyze the dynamics and to develop more control methods for this mechanical system. This is the main motivation of this study. In this paper, a set of suitable state variables are selected for the UHSTM system based on its nonlinear dynamics. It changes the UHSTM into a cascade nonlinear system. And then, the global stabilization control problem for the cascade system is concerned. Based on the structural characteristics of the cascade system, a positive-definite Lyapunov function is constructed. A control law is designed to guarantee the closed-loop control system to be Lyapunov stable. After that, the asymptotical stability of the control system is discussed by LaSalle's invariance principle. Moreover, the conditions that ensure the system to globally converge to the origin are presented. Finally, numerical examples verified the effectiveness of the presented theoretical results.

\section{Model and Nonlinear Dynamics Analysis of the UHSTM System}

Figure 1 shows the model of the UHSTM, where $m_{i}, L_{i}$, and $J_{i}$ are the mass, the length, and the moment of inertia of the $i$-th link, respectively, $(i=1,2) ; L_{c 1}$ is the distance from the spring joint to the COM of the first link, and $L_{c 2}$ is the distance from the active joint to the COM of the second link; $q_{i}(t)$ is the rotational angle of the $i$-th link $(i=1,2) ; F(t)$ is the input torque applied on the active joint; and $k$ is the elastic coefficient of the spring. Assume that the spring is fully relaxed when $q_{1}(t)=0$. It is not difficult to get the kinetic and potential energy of the system as

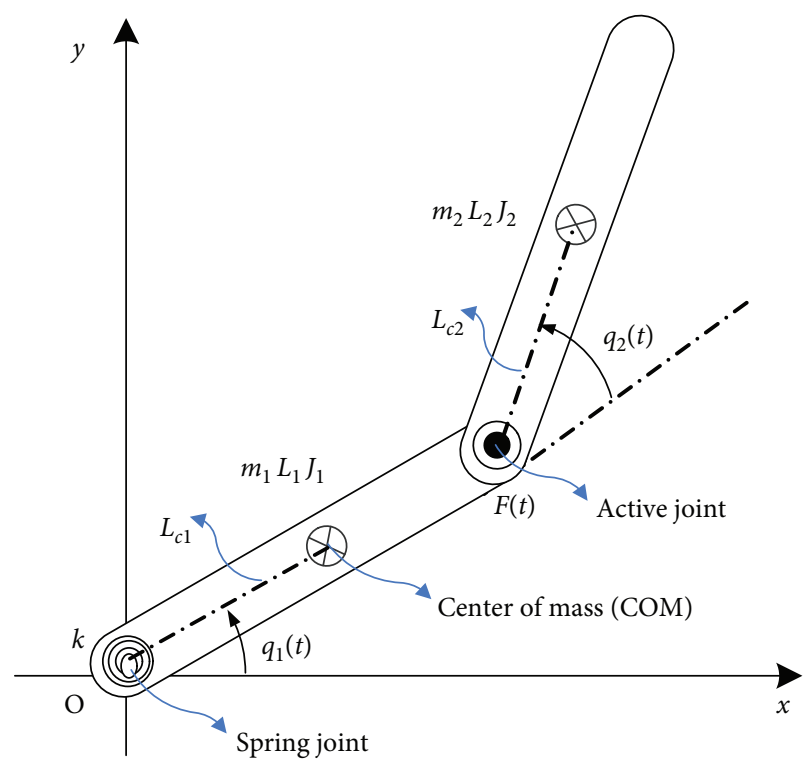

Figure 1: Model of underactuated horizontal spring-coupled twolink manipulators (UHSTM).

$$
\begin{aligned}
K(\mathbf{q}, \dot{\mathbf{q}}) & =\frac{1}{2} D_{11}\left(q_{2}\right) \dot{q}_{1}^{2}(t)+D_{12}\left(q_{2}\right) \dot{q}_{1}(t) \dot{q}_{2}(t)+\frac{1}{2} D_{22} \dot{q}_{2}^{2}(t), \\
P(\mathbf{q}) & =\frac{1}{2} k q_{1}^{2}(t),
\end{aligned}
$$

where $\mathbf{q}(t)=\left[q_{1}(t), q_{2}(t)\right]^{T}, \dot{\mathbf{q}}(t)=\mathrm{d} \mathbf{q}(t) / \mathrm{d} t$, and

$$
\begin{aligned}
D_{11}\left(q_{2}\right) & =\alpha_{1}+\alpha_{2}+2 \alpha_{3} \cos q_{2}(t), \\
D_{12}\left(q_{2}\right) & =D_{21}\left(q_{2}\right)=\alpha_{2}+\alpha_{3} \cos q_{2}(t), D_{22}=\alpha_{2}, \\
\alpha_{1} & =m_{1} L_{c 1}^{2}+m_{2} L_{1}^{2}+J_{1}, \\
\alpha_{2} & =m_{2} L_{c 2}^{2}+J_{2}, \\
\alpha_{3} & =m_{2} L_{1} L_{c 2} .
\end{aligned}
$$

We take the Lagrangian of the UHSTM system to be $L(\mathbf{q}, \dot{\mathbf{q}})=K(\mathbf{q}, \dot{\mathbf{q}})-P(\mathbf{q})$. The Euler-Lagrange motion equation of the system is obtained as

$$
\begin{aligned}
& \frac{\mathrm{d}}{\mathrm{d} t}\left[\frac{\partial L(\mathbf{q}, \dot{\mathbf{q}})}{\partial \dot{q}_{1}}\right]-\frac{\partial L(\mathbf{q}, \dot{\mathbf{q}})}{\partial q_{1}}=0, \\
& \frac{\mathrm{d}}{\mathrm{d} t}\left[\frac{\partial L(\mathbf{q}, \dot{\mathbf{q}})}{\partial \dot{q}_{2}}\right]-\frac{\partial L(\mathbf{q}, \dot{\mathbf{q}})}{\partial q_{2}}=F(t),
\end{aligned}
$$

where $F(t)$ is the control input applied to variable $q_{2}(t)$, which is for the actuated part. It is equivalent to the following form:

$$
\begin{aligned}
& {\left[\begin{array}{cc}
D_{11}\left(q_{2}\right) & D_{12}\left(q_{2}\right) \\
D_{21}\left(q_{2}\right) & D_{22}
\end{array}\right]\left[\begin{array}{l}
\ddot{q}_{1}(t) \\
\ddot{q}_{2}(t)
\end{array}\right]+\left[\begin{array}{l}
H_{1}(\mathbf{q}, \dot{\mathbf{q}}) \\
H_{2}(\mathbf{q}, \dot{\mathbf{q}})
\end{array}\right]} \\
& \quad+\left[\begin{array}{c}
k q_{1}(t) \\
0
\end{array}\right]=\left[\begin{array}{c}
0 \\
F(t)
\end{array}\right],
\end{aligned}
$$

where 


$$
\begin{aligned}
& H_{1}(\mathbf{q}, \dot{\mathbf{q}})=-\alpha_{3}\left[2 \dot{q}_{1}(t)+\dot{q}_{2}(t)\right] \dot{q}_{2}(t) \sin q_{2}(t), \\
& H_{2}(\mathbf{q}, \dot{\mathbf{q}})=\alpha_{3} \dot{q}_{1}^{2}(t) \sin q_{2}(t) .
\end{aligned}
$$

It is clear that (3a) and (3b) is a complicated nonlinear system. In order to make the control design of the system be easy to solve, it is necessary to choose a set of state variables to change the system into the state space form. Note that $q_{2}(t)$ and $\dot{q}_{2}(t)$ are actuated variables of the UHSTM system. As the partial feedback linearization method developed in [20], we choose them to be two state variables of the system. In addition, it follows from (3a) that the first derivative of $\partial L(\mathbf{q}, \dot{q}) / \partial \dot{q}_{1}$ is equal to $\partial L(\mathbf{q}, \dot{q}) / \partial q_{1}$. A simple calculation shows that $\partial L(\mathbf{q}, \dot{q}) / \partial q_{1}$ is not related to $\ddot{q}$. So, we choose it as the third state variable of the system. Based on the expression of $\partial L(\mathbf{q}, \dot{q}) / \partial \dot{q}_{1}$ and $D_{11}\left(q_{2}\right)>0$, the fourth state variable of the system is chosen to be $q_{1}(t)+\xi\left(q_{2}\right)$ in order to make the dynamic structure of the system in the state space be simple, where

$$
\begin{aligned}
\xi\left(q_{2}\right)= & \int_{0}^{q_{2}(t)} \frac{D_{12}(\nu)}{D_{11}(v)} \mathrm{d} \nu=\int_{0}^{q_{2}(t)} \frac{\alpha_{2}+\alpha_{3} \cos \nu}{\alpha_{1}+\alpha_{2}+2 \alpha_{3} \cos \nu} \mathrm{d} \nu \\
= & \frac{q_{2}(t)}{2}+\frac{\alpha_{2}-\alpha_{1}}{\sqrt{\left(\alpha_{1}+\alpha_{2}\right)^{2}-4 \alpha_{3}^{2}}} \\
& \cdot \arctan \left(\sqrt{\frac{\alpha_{1}+\alpha_{2}-2 \alpha_{3}}{\alpha_{1}+\alpha_{2}+2 \alpha_{3}}} \tan \frac{q_{2}(t)}{2}\right) .
\end{aligned}
$$

In summary, the state variables of the UHSTM system are chosen to be

$$
\left\{\begin{array}{l}
z_{1}(t)=q_{1}(t)+\xi\left(q_{2}\right), \\
z_{2}(t)=\frac{\partial L(\mathbf{q}, \dot{\mathbf{q}})}{\partial \dot{q}_{1}}=D_{11}\left(q_{2}\right) \dot{q}_{1}(t)+D_{12}\left(q_{2}\right) \dot{q}_{2}(t), \\
z_{3}(t)=q_{2}(t), \\
z_{4}(t)=\dot{q}_{2}(t) .
\end{array}\right.
$$

Let $\mathbf{y}(t)=\left[q_{1}(t), q_{2}(t), \dot{q}_{1}(t), \dot{q}_{2}(t)\right]^{T}$ and $\mathbf{z}(t)=\left[z_{1}(t)\right.$, $\left.z_{2}(t), z_{3}(t), z_{4}(t)\right]^{T}$. It is easy to get

$$
\begin{aligned}
\left|\frac{\partial \mathbf{z}(t)}{\partial \mathbf{y}(t)}\right| & =\|\left[\begin{array}{cccc}
1 & \frac{D_{12}\left(q_{2}\right)}{D_{11}\left(q_{2}\right)} & 0 & 0 \\
0 & -\alpha_{3} \sin q_{2}(t)\left(2 \dot{q}_{1}+\dot{q}_{2}\right) & D_{11}\left(q_{2}\right) & D_{12}\left(q_{2}\right) \\
0 & 1 & 0 & 0 \\
0 & 0 & 0 & 1
\end{array}\right] \\
& =-D_{11}\left(q_{2}\right)<0 .
\end{aligned}
$$

It means that the transformation from $\mathbf{y}(t)$ to $\mathbf{z}(t)$ is homeomorphous. From (7), we have

$$
\begin{aligned}
& \dot{z}_{1}(t)=\dot{q}_{1}(t)+\frac{d \xi\left(q_{2}\right)}{d q_{2}} \dot{q}_{2}(t)=\frac{D_{11}\left(q_{2}\right) \dot{q}_{1}(t)+D_{12}\left(q_{2}\right) \dot{q}_{2}(t)}{D_{11}\left(q_{2}\right)}=\frac{z_{2}(t)}{D_{11}\left(z_{3}\right)}, \\
& \dot{z}_{2}(t)=\frac{\mathrm{d}}{\mathrm{d} t}\left[\frac{\partial L(\mathbf{q}, \dot{\mathbf{q}})}{\partial \dot{q}_{1}}\right]=\frac{\partial L(\mathbf{q}, \dot{\mathbf{q}})}{\partial q_{1}}=-k q_{1}(t)=-k\left[z_{1}(t)-\xi\left(q_{2}\right)\right], \\
& \dot{z}_{3}(t)=\dot{q}_{2}(t)=z_{4}(t), \\
& \dot{z}_{4}(t)=\ddot{q}_{2}(t) .
\end{aligned}
$$

These give the state space equation of the UHSTM system as

$$
\left\{\begin{array}{l}
\dot{z}_{1}(t)=\frac{z_{2}(t)}{D_{11}\left(z_{3}\right)} \\
\dot{z}_{2}(t)=-k z_{1}(t)+k \xi\left(z_{3}\right) \\
\dot{z}_{3}(t)=z_{4}(t) \\
\dot{z}_{4}(t)=\tau(t)
\end{array}\right.
$$

where $\tau(t)=\ddot{q}_{2}(t)$ is the control input of system (10). From (4), it is not difficult to get

$$
\begin{aligned}
& F(t)=D_{21}\left(q_{2}\right) \ddot{q}_{1}(t)+D_{22} \ddot{q}_{2}(t)+H_{2}(\mathbf{q}, \dot{\mathbf{q}}), \\
& D_{11}\left(q_{2}\right) \ddot{q}_{1}(t)+D_{12} \ddot{q}_{2}(t)+H_{1}(\mathbf{q}, \dot{\mathbf{q}})+k q_{1}(t)=0 .
\end{aligned}
$$

Eliminating the term $\ddot{q}_{1}(t)$ from the above equations and considering $\tau(t)=\ddot{q}_{2}(t)$ yield

$$
F(t)=H_{2}(\mathbf{q}, \dot{\mathbf{q}})-\frac{D_{21}\left(q_{2}\right)\left[H_{1}(\mathbf{q}, \dot{\mathbf{q}})+k q_{1}(t)\right]}{D_{11}\left(q_{2}\right)}+\frac{\Delta\left(q_{2}\right)}{D_{11}\left(q_{2}\right)} \tau(t),
$$

where $\Delta\left(q_{2}\right)=D_{11}\left(q_{2}\right) D_{22}-D_{12}\left(q_{2}\right) D_{21}\left(q_{2}\right)>0$. This is the relationship between the control inputs $F(t)$ and $\tau(t)$.

The main concern of this paper is to design the control law $F(t)$ that globally stabilizes the UHSTM at $\mathbf{y}(t)=0$. Note that $\mathbf{y}(t)=\mathbf{0}$ is equivalent to $\mathbf{z}(t)=0$. So, the discussed 
stabilizing control objective is achieved so long as system (10) is globally stabilized at $\mathbf{z}(t)=0$.

\section{Design of Stabilizing Control Law}

In this section, the stabilizing control of (10) at $\mathbf{z}(t)=0$ is discussed. A control law $\tau(t)$ is designed by using Lyapunov stability theory. Note that (10) is a cascade nonlinear system. Based on the structural characteristics of this system, a function is constructed to be

$$
V(\mathbf{z})=\frac{\beta_{1}}{2} W^{2}(\mathbf{z})+\frac{\beta_{2}}{2} z_{3}^{2}(t)+\frac{\beta_{3}}{2} z_{4}^{2}(t),
$$

where $\beta_{i}>0(i=1,2,3)$ are constants, and

$$
W(\mathbf{z})=k z_{1}(t)\left[\frac{z_{1}(t)}{2}-\xi\left(z_{3}\right)\right]+\frac{z_{2}^{2}(t)}{2 D_{11}\left(z_{3}\right)} .
$$

Note that $V(\mathbf{z})$ is a nonnegative function. It is easy to verify that $V(\mathbf{z})=0$ when $\mathbf{z}(t)=0$. In addition, $V(\mathbf{z})=0$ gives $W(\mathbf{z})=0, \quad z_{3}(t)=0, \quad$ and $\quad z_{4}(t)=0$ because $\beta_{i}(i=1,2,3)$ are positive constants. In this case, it follows from (14) that

$$
W(\mathbf{z})=\frac{k z_{1}^{2}(t)}{2}+\frac{z_{2}^{2}(t)}{2 D_{11}(0)}=0 .
$$

Equation (15) means that $z_{1}(t)=0$ and $z_{2}(t)=0$ since $k>0$ and $D_{11}(0)>0$. So, we get that $\mathbf{z}(t)=0$ when $V(\mathbf{z})=0$. In summary, $V(\mathbf{z})=0$ is equivalent to $\mathbf{z}(t)=0$. Thus, $V(\mathbf{z})$ is a typical positive-definite Lyapunov function for system (10). Differentiating $W(\mathbf{z})$ along (10) gives

$$
\begin{aligned}
\frac{\mathrm{d} W(\mathbf{z})}{\mathrm{d} t}= & \frac{\mathrm{d} W(\mathbf{z})}{\mathrm{d} z_{1}} \frac{\mathrm{d} z_{1}(t)}{\mathrm{d} t}+\frac{\mathrm{d} W(\mathbf{z})}{\mathrm{d} z_{2}(t)} \frac{\mathrm{d} z_{2}(t)}{\mathrm{d} t}+\frac{\mathrm{d} W(\mathbf{z})}{\mathrm{d} z_{3}} \frac{\mathrm{d} z_{3}(t)}{\mathrm{d} t} \\
= & k\left[z_{1}(t)-\xi\left(z_{3}\right)\right] \frac{z_{2}(t)}{D_{11}\left(z_{3}\right)}+\frac{z_{2}(t)}{D_{11}\left(z_{3}\right)}\left[-k z_{1}(t)+k \xi\left(z_{3}\right)\right] \\
& +\left[\frac{\alpha_{3} z_{2}^{2}(t) \sin z_{3}(t)}{D_{11}^{2}\left(z_{3}\right)}-\frac{k z_{1}(t) D_{12}\left(z_{3}\right)}{D_{11}\left(z_{3}\right)}\right] z_{4}(t) \\
= & \Lambda(\mathbf{z}) z_{4}(t),
\end{aligned}
$$

where

$$
\Lambda(\mathbf{z})=\frac{\alpha_{3} z_{2}^{2}(t) \sin z_{3}(t)-k z_{1}(t) D_{12}\left(z_{3}\right) D_{11}\left(z_{3}\right) .}{D_{11}^{2}\left(z_{3}\right)} .
$$

It follows from (10), (13), and (16) that

$$
\begin{aligned}
\frac{\mathrm{d} V(\mathbf{z})}{\mathrm{d} t} & =\beta_{1} W(\mathbf{z}) \frac{\mathrm{d} W(\mathbf{z})}{\mathrm{d} t}+\beta_{2} z_{3}(t) z_{4}(t)+\beta_{3} z_{4}(t) \tau(t) \\
& =\left[\beta_{1} W(\mathbf{z}) \Lambda(\mathbf{z})+\beta_{2} z_{3}(t)+\beta_{3} \tau(t)\right] z_{4}(t) .
\end{aligned}
$$

The control law is designed to be

$$
\tau(t)=\frac{-\beta_{1} W(\mathbf{z}) \Lambda(\mathbf{z})-\beta_{2} z_{3}(t)-\gamma z_{4}(t)}{\beta_{3}},
$$

where $\gamma>0$ is a constant. Combining (18) and (19) gives

$$
\frac{\mathrm{d} V(\mathbf{z})}{\mathrm{d} t}=-\gamma z_{4}^{2}(t) \leq 0 .
$$

Since $V(\mathbf{z})$ is positive definite and $\dot{V}(\mathbf{z}) \leq 0$, closed-loop control systems (10) and (19) are Lyapunov stable.

\section{Asymptotical Stability Analysis of the Closed- Loop Control System}

In order to get the final stabilizing state of closed-loop systems (10) and (19), the asymptotical stability of the system is analyzed by using LaSalle's invariance principle. Letting $\dot{V}(\mathbf{z}) \equiv 0$ gives $x_{4}(t)=0$. It follows from the third and fourth equations of (10) that $z_{3}(t)=z_{3}^{*}$ and $\tau(t)=0$, where $z_{3}^{*}$ is a constant. The further stability analysis results are summarized in the following two theorems.

Theorem 1. If $z_{3}(t)=z_{3}^{*}=0$, then $\dot{V}(\mathbf{z}) \equiv 0$ gives $\mathbf{z}(t)=0$.

Proof. From $z_{3}(t)=0, z_{4}(t)=0, \tau(t)=0$, and (19), it is easy to obtain $z_{1}(t) W(\mathbf{z})=0$. Thus, $z_{1}(t)=0$ or $W(\mathbf{z})=0$. If $z_{1}(t)=0$, then the first equation of (10) gives $z_{2}(t)=0$. If $W(\mathbf{z})=0$, then combining $z_{3}(t)=0$, (14), and (15) gives $z_{1}(t)=z_{2}(t)=0$. So, $\dot{V}(\mathbf{z}) \equiv 0$ gives $\mathbf{z}(t)=0$. The proof is completed.

Theorem 2. If $z_{3}(t)=z_{3}^{*} \neq 0$ and $\alpha_{2} \neq \alpha_{3}$, then $\dot{V}(\mathbf{z}) \equiv 0$ gives $z_{2}(t)=0, z_{1}(t)=\xi\left(z_{3}^{*}\right)$, and

$$
\frac{\beta_{1} k^{2} \xi^{3}\left(z_{3}^{*}\right)\left[\alpha_{2}+\alpha_{3} \cos z_{3}^{*}\right]}{2\left[\alpha_{1}+\alpha_{2}+\alpha_{3} \cos z_{3}^{*}\right]}+\beta_{2} z_{3}^{*}=0 .
$$

Proof. Combining $z_{4}(t)=0, \tau(t)=0$, (17), and (19) yields $\beta_{1} W(\mathbf{z})\left[\alpha_{3} z_{2}^{2}(t) \sin z_{3}^{*}-k z_{1}(t) D_{12}\left(z_{3}^{*}\right) D_{11}\left(z_{3}^{*}\right)\right]+\beta_{2} D_{11}^{2}\left(z_{3}^{*}\right) z_{3}^{*}=0$.

Since $\dot{V}(\mathbf{z}) \equiv 0$ means that $V(\mathbf{z})$ equals to a constant, it follows from $z_{3}(t)=z_{3}^{*}, \quad z_{4}(t)=0$, and (13) that $W(\mathbf{z})=W^{*}$, where $W^{*}$ is a constant. From $D_{11}\left(z_{3}^{*}\right)>0$, $z_{3}^{*} \neq 0$, and (22), it is easy to get that $W^{*} \neq 0$. As a result, (22) gives

$$
\alpha_{3} z_{2}^{2}(t) \sin z_{3}^{*}-k z_{1}(t) D_{12}\left(z_{3}^{*}\right) D_{11}\left(z_{3}^{*}\right)=C,
$$

where $C$ is a non-zero constant. Differentiating (23) along (10) yields

$$
\left\{2 \alpha_{3}\left[z_{1}(t)-\xi\left(z_{3}^{*}\right)\right] \sin z_{3}^{*}+D_{12}\left(z_{3}^{*}\right)\right\} z_{2}(t)=0 .
$$

We continue carrying out the analysis in the following two situations.

Case 1. $\mathbf{z}_{2}(t) \neq 0$ : it follows from (24) that

$$
2 \alpha_{3}\left[z_{1}(t)-\xi\left(z_{3}^{*}\right)\right] \sin z_{3}^{*}+D_{12}\left(z_{3}^{*}\right)=0 .
$$

If $\sin z_{3}^{*}=0$, then (25) gives $D_{12}\left(z_{3}^{*}\right)=0$, i.e., $\alpha_{2}+\alpha_{3} \cos z_{3}^{*}=0$. As a result, $\sin z_{3}^{*}=0$ and $\alpha_{2}+\alpha_{3} \cos z_{3}^{*}=0$ are contradictory because $\alpha_{2}>0, \alpha_{3}>0$, and $\alpha_{2} \neq \alpha_{3}$. Thus, it concludes that $\sin z_{3}^{*} \neq 0$. So, (25) gives 


$$
z_{1}(t)=\frac{-D_{12}\left(z_{3}^{*}\right)}{2 \alpha_{3} \sin z_{3}^{*}}+\xi\left(z_{3}^{*}\right),
$$

which is a constant. It follows from the first equation of (10) that $z_{2}(t)=0$. This is a contradiction.

Case 2. $\mathbf{z}_{2}(t)=0$ : the first and second equations of (10) give that $z_{1}(t)=z_{1}^{*}$ and $z_{1}^{*}=\xi\left(z_{3}^{*}\right)$, where $z_{1}^{*}$ is a constant. Substituting $z_{2}(t)=0$ and $z_{1}^{*}=\xi\left(z_{3}^{*}\right)$ into (14) yields $W(\mathbf{z})=-k \xi^{2}\left(z_{3}^{*}\right) / 2$. Combining $W(\mathbf{z})$ and (22) gives

$$
\frac{\beta_{1} k^{2} D_{12}\left(z_{3}^{*}\right) \xi^{3}\left(z_{3}^{*}\right)}{2 D_{11}\left(z_{3}^{*}\right)}+\beta_{2} z_{3}^{*}=0 .
$$

It is equivalent to equation (21). The proof is completed. Based on the analysis results in Theorem 1 and Theorem 2, $\dot{V}(\mathbf{z}) \equiv 0$ gives

$$
\Omega=\left\{\mathbf{z}(t)=0 \text { or } \mathbf{z}(t)=\left[\xi\left(z_{3}^{*}\right), 0, z_{3}^{*}, 0\right]\right\},
$$

where $z_{3}^{*}$ is the solution of (21). According to LaSalle's invariance theorem [27], closed-loop control systems (10) and (19) asymptotically converge to the largest invariant set contained in $\Omega$.

In order to clearly describe the stabilizing state of the control system, it is necessary to solve (21). Note that (21) is a complicated transcendental equation. It is difficult to obtain an analytical expression for $z_{3}^{*}$. To solve this problem, a condition on the control parameters $\beta_{1}$ and $\beta_{2}$ is presented, which ensures (21) has only one solution for $z_{3}^{*}$. The main result is given in the following theorem.

Theorem 3. If the control parameters $\beta_{1}$ and $\beta_{2}$ satisfy

$$
\beta_{2}>\frac{k^{2} \alpha_{3}\left|\alpha_{2}-\alpha_{1}\right|\left(\alpha_{2}+\alpha_{3}\right)^{3} \pi^{3}}{2\left(\alpha_{1}+\alpha_{2}-2 \alpha_{3}\right)^{5}} \beta_{1}
$$

then equation (21) has only one solution $z_{3}^{*}=0$. And thus, closed-loop control systems (10) and (19) asymptotically converge to $\mathbf{z}(t)=0$.

Proof. Note that $z_{3}(t)=q_{2}(t)$ stands for the angle position of the second link of the UHSTM (see Figure 1). It is a cyclic variable with a period $2 \pi$. So, we can assume that $-\pi \leq z_{3}(t)<\pi(\bmod 2 \pi)$ in this paper. Based on $(21)$, an auxiliary function is designed to be

$$
f(x)=\frac{\beta_{1} g(x)}{\alpha_{1}+\alpha_{2}+2 \alpha_{3} \cos x}+\beta_{2} x, \quad x \in[-\pi, \pi),
$$

where

$$
\begin{aligned}
& g(x)=\frac{k^{2} \xi^{3}(x)\left[\alpha_{2}+\alpha_{3} \cos x\right]}{2}, \\
& \xi(x)=\int_{0}^{x} \frac{\alpha_{2}+\alpha_{3} \cos \nu}{\alpha_{1}+\alpha_{2}+2 \alpha_{3} \cos \nu} \mathrm{d} \nu .
\end{aligned}
$$

It is not difficult to obtain

$$
\begin{aligned}
& |\xi(x)| \leq \frac{\alpha_{2}+\alpha_{3}}{\alpha_{1}+\alpha_{2}-2 \alpha_{3}}|x| \leq \frac{\left(\alpha_{2}+\alpha_{3}\right) \pi}{\alpha_{1}+\alpha_{2}-2 \alpha_{3}}, \\
& \frac{\mathrm{d} g(x)}{\mathrm{d} x}=\frac{k^{2} \xi^{2}(x)}{2}\left[\frac{3\left(\alpha_{2}+\alpha_{3} \cos x\right)^{2}}{\alpha_{1}+\alpha_{2}-2 \alpha_{3} \cos x}-\alpha_{3} \xi(x) \sin x\right] .
\end{aligned}
$$

Combining (30) and (33) yields

$$
\begin{aligned}
\frac{\mathrm{d} f(x)}{\mathrm{d} x} & =\beta_{1} \frac{(\mathrm{d} g(x) / \mathrm{d} x)\left(\alpha_{1}+\alpha_{2}+2 \alpha_{3} \cos x\right)+2 \alpha_{3} g(x) \sin x}{\left(\alpha_{1}+\alpha_{2}+2 \alpha_{3} \cos x\right)^{2}}+\beta_{2} \\
& =\beta_{2}+\frac{\beta_{1} k^{2}}{2}\left[\frac{\alpha_{3}\left(\alpha_{2}-\alpha_{1}\right) \xi^{3}(x) \sin x}{\left(\alpha_{1}+\alpha_{2}+2 \alpha_{3} \cos x\right)^{2}}+\frac{3 \xi^{2}(x)\left(\alpha_{2}+\alpha_{3} \cos x\right)^{2}}{\left(\alpha_{1}+\alpha_{2}+2 \alpha_{3} \cos x\right)^{2}}\right] \\
& \geq \beta_{2}+\frac{\beta_{1} k^{2} \alpha_{3}\left(\alpha_{2}-\alpha_{1}\right) \xi^{3}(x) \sin x}{2\left(\alpha_{1}+\alpha_{2}+2 \alpha_{3} \cos x\right)^{2}} .
\end{aligned}
$$

It follows from (32) that

$$
\left|\frac{\left(\alpha_{2}-\alpha_{1}\right) \xi^{3}(x) \sin x}{\left(\alpha_{1}+\alpha_{2}+2 \alpha_{3} \cos x\right)^{2}}\right| \leq \frac{\left|\alpha_{2}-\alpha_{1}\right||\xi(x)|^{3}}{\left(\alpha_{1}+\alpha_{2}-2 \alpha_{3}\right)^{2}} \leq \frac{\left|\alpha_{2}-\alpha_{1}\right|\left(\alpha_{2}+\alpha_{3}\right)^{3} \pi^{3}}{\left(\alpha_{1}+\alpha_{2}-2 \alpha_{3}\right)^{5}} .
$$

Combining (29), (34), and (35) yields $\mathrm{d} f(x) / \mathrm{d} x>0$. It means that $f(x)$ is a strictly monotonically increasing function during $x \in[-\pi, \pi)$. By the fact that $f(0)=0$, it is easy to conclude that $f(x)=0$ has only one solution $x=0$.
In other words, condition (29) ensures that (21) has only one solution $z_{3}^{*}=0$. Substituting $z_{3}^{*}=0$ into (28) yields that $\Omega$ contains only one element $\mathbf{z}(t)=0$. So, systems (10) and (19) asymptotically converge to $\mathbf{z}(t)=0$. The proof is completed.

From the above statements, we get that the control law $\tau(t)$ in (19) globally stabilizes system (10) at $\mathbf{z}(t)=0$ so as to both conditions, $\alpha_{2} \neq \alpha_{3}$ and (29), are satisfied. As a result, the control law $F(t)$ obtained by substituting $\tau(t)$ into (12) globally stabilizes the UHSTM at $\mathbf{y}(t)=0$ under these conditions. 
TABLE 1: Parameters of the underactuated horizontal spring-coupled two-link manipulator (UHSTM).

\begin{tabular}{|c|c|c|c|c|}
\hline$m_{1}(\mathrm{~kg})$ & $m_{2}(\mathrm{~kg})$ & $L_{1}(\mathrm{~m})$ & $L_{2}(\mathrm{~m})$ & $l_{1}(\mathrm{~m})$ \\
\hline 1 & 1 & 1 & 2 & 0.5 \\
\hline$l_{2}(\mathrm{~m})$ & $J_{1}\left(\mathrm{~kg} \mathrm{~m}^{2}\right)$ & $J_{2}\left(\mathrm{~kg} \mathrm{~m}^{2}\right)$ & $k(\mathrm{~N} / \mathrm{m})$ & \\
\hline
\end{tabular}

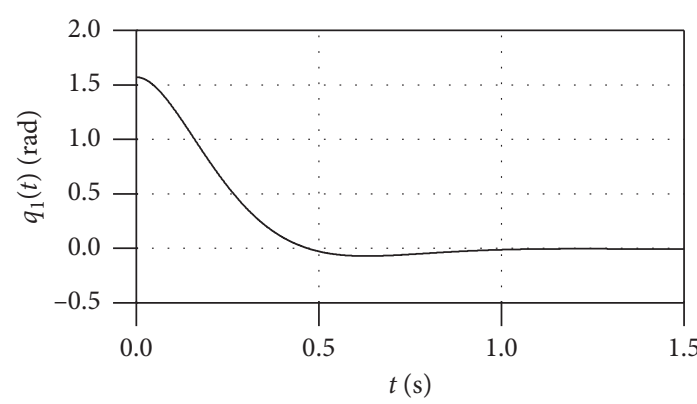

(a)

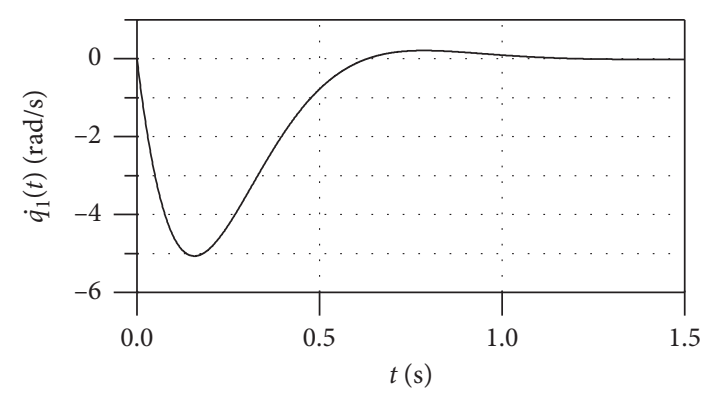

(c)

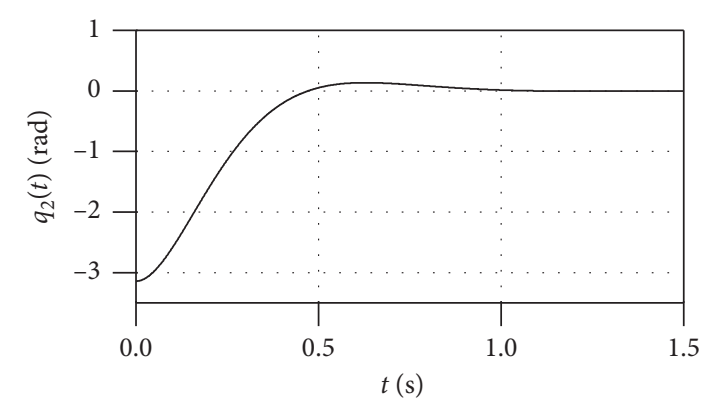

(b)

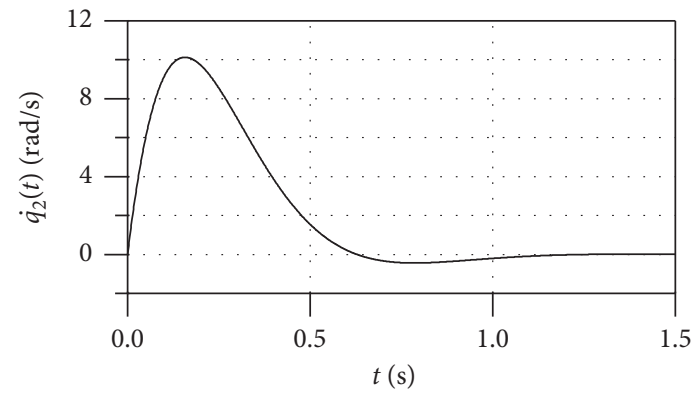

(d)

FIGURE 2: Time response of $q_{i}(t)$ and $\dot{q}_{i}(t)(i=1,2)$ with initial condition (37).

\section{Numerical Examples}

This section presents numerical examples to verify the effectiveness of the above theoretical results.

The physical parameters of the UHSTM presented in [26] were chosen for simulation. They are listed in Table 1. The control parameters in (19) were chosen to be $\beta_{1}=5$, $\beta_{2}=5, \beta_{3}=0.1$, and $\gamma=1$. A simple calculation gives

$$
\begin{aligned}
\alpha_{1} & =1.333, \\
\alpha_{2} & =1.33, \\
\alpha_{3} & =1, \\
\frac{k^{2} \alpha_{3}\left|\alpha_{2}-\alpha_{1}\right|\left(\alpha_{2}+\alpha_{3}\right)^{3} \pi^{3}}{2\left(\alpha_{1}+\alpha_{2}-2 \alpha_{3}\right)^{5}} \beta_{1} & =0.2296 .
\end{aligned}
$$

So, both conditions, $\alpha_{2} \neq \alpha_{3}$ and (29), are satisfied. The simulation results of the UHSTM with an initial condition,

$$
\left[q_{1}(0), q_{2}(0), \dot{q}_{1}(0), \dot{q}_{2}(0)\right]^{T}=\left[\frac{\pi}{2},-\pi, 0,0\right]^{T},
$$

are shown in Figure 2. Note that the UHSTM is quickly stabilized at the origin. For the sake of comparison, we also carried out the simulation for the UHSTM with the same initial condition as in [26], i.e.,

$$
\left[q_{1}(0), q_{2}(0), \dot{q}_{1}(0), \dot{q}_{2}(0)\right]^{T}=[-1,2,0,0]^{T} .
$$

The simulation results with (38) show that our method is still effective (see Figure 3). The stabilizing time is less than 1.5 seconds. In contrast, the stabilizing time of the UHSTM in [26] is greater than 5 seconds. This shows the superiority of our presented method.

In order to verify the practicality of our method in a real environment, the simulations were carried out by considering parameter uncertainties $( \pm 5 \%$ of their nominal values for $m_{i}, L_{c i}$, and $J_{i}, i=1,2$ ), white noises (peak value: \pm 0.15 ) in measurements, and the viscous friction disturbances in the joints. The results show that our method is still effective under these conditions. We give the simulation results in Figure 4 for the typical case, where $m_{1}, L_{c 1}$, and $J_{1}$ are $5 \%$ smaller than their nominal values; $m_{2}, L_{c 2}$, and $J_{2}$ are $5 \%$ larger than their nominal values; the peak value of white noise is \pm 0.1 ; and the friction disturbances are $f_{v 1}(t)=$ $-0.15 \dot{q}_{1}(t)$ and $f_{v 2}(t)=-0.25 \dot{q}_{1}(t)$ in the spring joint and the active joint, respectively. Note that the UHSTM can still be stabilized at the origin. The robustness of our method is good. 


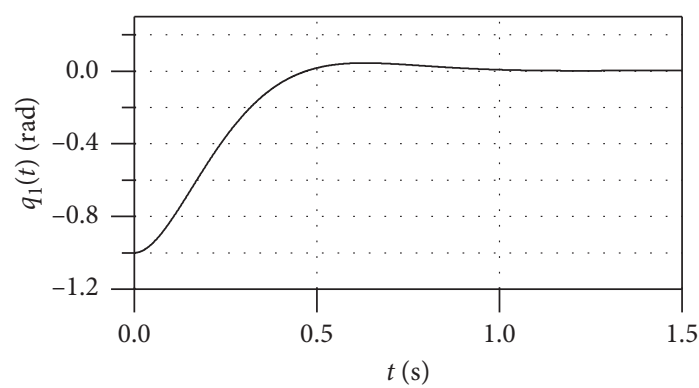

(a)

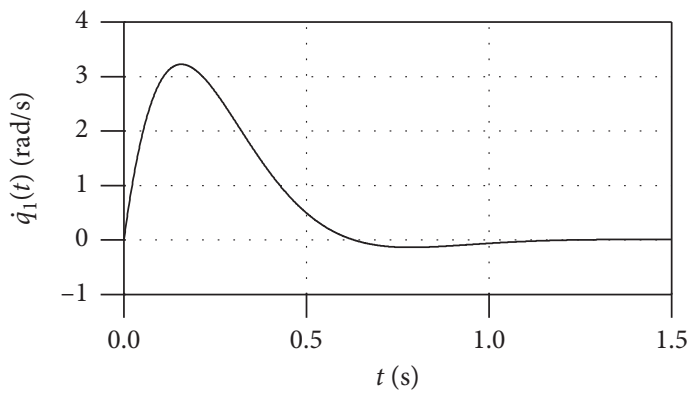

(c)

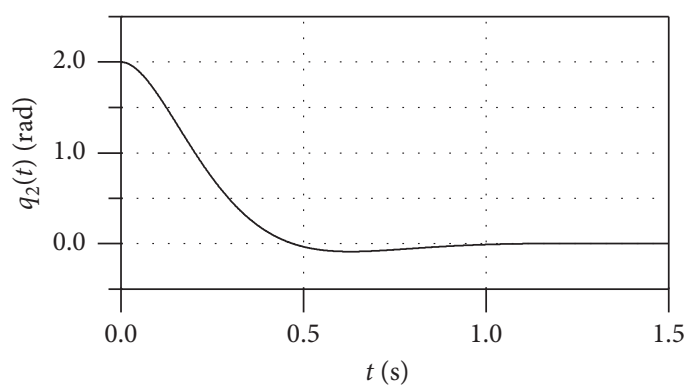

(b)

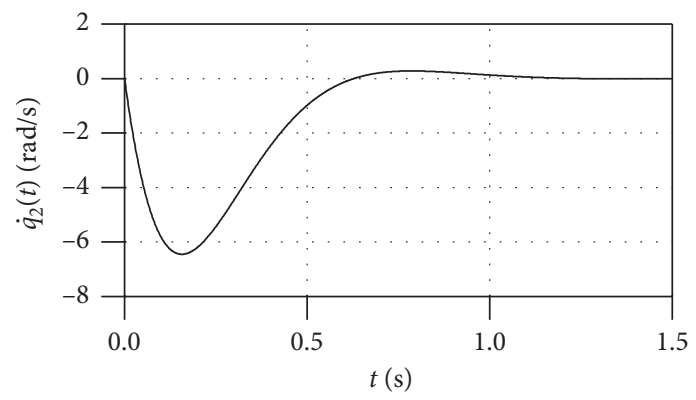

(d)

Figure 3: Time response of $q_{i}(t)$ and $\dot{q}_{i}(t)(i=1,2)$ with initial condition (38).

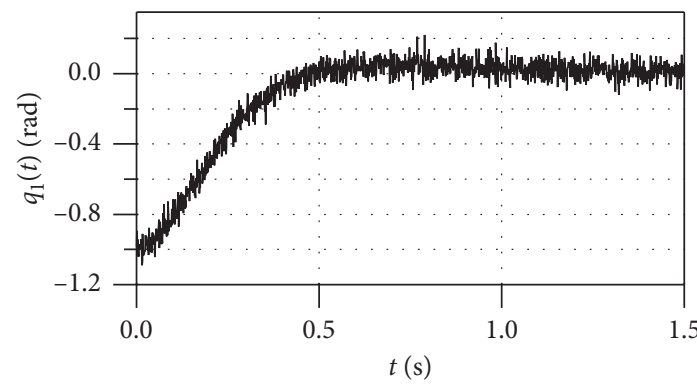

(a)

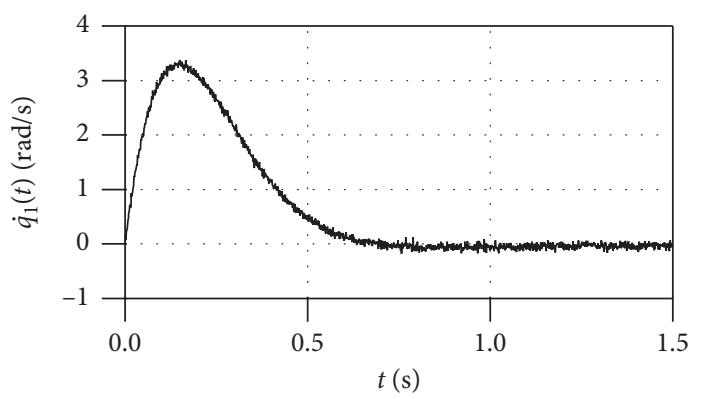

(c)

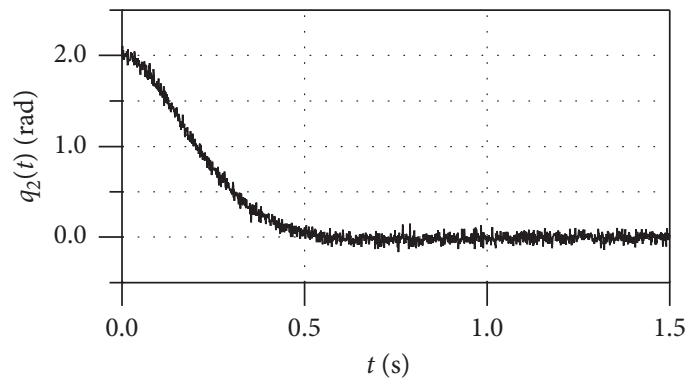

(b)

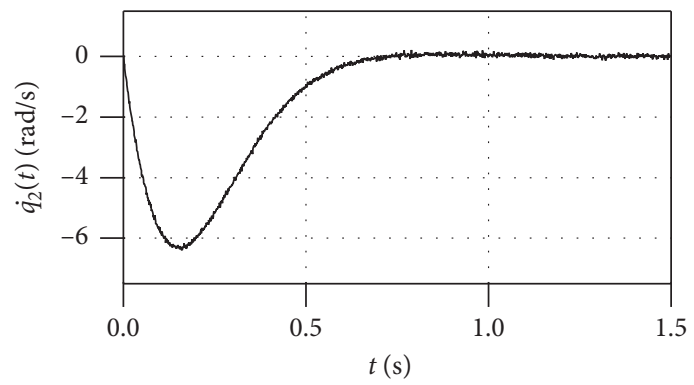

(d)

FIgURE 4: Time response of $q_{i}(t)$ and $\dot{q}_{i}(t)(i=1,2)$ with initial condition (38) when parameter uncertainties, white noises, and friction exist.

\section{Conclusions}

This paper concerned the nonlinear dynamics analysis and the global stabilization control of a 2 -DOF underactuated system called UHSTM. A set of suitable state variables were constructed that change the system into a cascade nonlinear system. Then, a stabilizing control law was designed for the cascade system based on the Lyapunov theory. After that, the conditions were presented in order to guarantee the global asymptotical stability of the closed-loop control system at the origin. Numerical examples verified the effectiveness of the method. In the future, we will further study how to 
extend the method to stabilize the underactuated horizontal multilink manipulator and other underactuated nonlinear systems.

\section{Data Availability}

The data used to support the findings of this study are available from the corresponding author upon request.

\section{Conflicts of Interest}

The authors declare that they have no conflicts of interest.

\section{Acknowledgments}

This work was supported in part by the Natural Science Foundation Program of Shandong Province under Grant no. ZR2019YQ28, the Development Plan of Youth Innovation Team of University in Shandong Province under Grant no. 2019KJN007, and the National Natural Science Foundation of China under Grant nos. 61773193, 61803194, 61877033 and 61903171.

\section{References}

[1] I. Fonseca, L. Goes, N. Seito, M. Duarte, and É. Oliveira, "Attitude dynamics and control of a spacecraft like a robotic manipulator when implementing on-orbit servicing," Acta Astronautica, vol. 137, pp. 490-497, 2017.

[2] R. Mei and C. Yu, "Adaptive neural output feedback control for uncertain robot manipulators with input saturation," Complexity, vol. 2017, Article ID 7413642, 12 pages, 2017.

[3] M. Llama, A. Flores, V. Santibáez, and R. Campa, "Global convergence of a decentralized adaptive fuzzy control for the motion of robot manipulators: application to the mitsubishi PA10-7CE as a case of study," Journal of Intelligent \& Robotic Systems, vol. 82, no. 3-4, pp. 363-377, 2016.

[4] W. Yu and J. Rosen, "Neural PID control of robot manipulators with application to an upper limb exoskeleton," IEEE Transactions on Cybernetics, vol. 43, no. 2, pp. 673-684, 2013.

[5] Y. Liu and H. Yu, "A survey of underactuated mechanical systems," IET Control Theory \& Applications, vol. 7, no. 7, pp. 921-935, 2013.

[6] S. Krafes, Z. Chalh, and A. Saka, "A review on the control of second order underactuated mechanical systems," Complexity, vol. 2018, Article ID 9573514, 17 pages, 2018.

[7] A. Zhang, J. She, J. Qiu, C. Yang, and F. Alsaadi, "Design of motion trajectory and tracking control for underactuated cart-pendulum system," International Journal of Robust Nonlinear Control, vol. 29, no. 9, Article ID 2458C2470, 2019.

[8] N. Sun, Y. Wu, Y. Fang, H. Chen, and B. Lu, "Nonlinear continuous global stabilization control for underactuated RTAC systems: design, analysis, and experimentation," IEEE/ ASME Transactions on Mechatronics, vol. 22, no. 2, pp. 1104-1115, 2017.

[9] N. Sun, Y. Wu, H. Chen, and Y. Fang, "Antiswing cargo transportation of underactuated tower crane systems by a nonlinear controller embedded with an integral term," IEEE Transactions on Automation Science and Engineering, vol. 16, no. 3, pp. 1387-1398, 2019.

[10] H. Chen and N. Sun, "Nonlinear control of underactuated systems subject to both actuated and unactuated state constraints with experimental verification," IEEE Transactions on Industrial Electronics, 2019.

[11] J. She, A. Zhang, X. Lai, and M. Wu, "Global stabilization of 2DOF underactuated mechanical systemsłan equivalent-inputdisturbance approach," Nonlinear Dynamics, vol. 69, no. 1-2, pp. 495-509, 2012.

[12] G. Oriolo and Y. Nakamura, "Control of mechanical systems with second-order nonholonomic constraints: underactuated manipulators," in Proceedings of the 30th IEEE Conference on Decision and Control, pp. 2398-2403, Brighton, UK, December 1991.

[13] X. Xin, J. She, and Y. Liu, "A unified solution to swing-up control for $n$-link planar robot with single passive joint based on virtual composite links and passivity," Nonlinear Dynamics, vol. 67, no. 2, pp. 909-923, 2012.

[14] X. Lai, J. She, S. Yang, and M. Wu, "Comprehensive unified control strategy for underactuated two-link manipulators," IEEE Transactions on Systems, Man, and Cybernetics-Part B: Cybernetics, vol. 39, no. 2, pp. 389-398, 2009.

[15] A. Zhang, X. Lai, M. Wu, and J. She, "Stabilization of underactuated two-link gymnast robot by using trajectory tracking strategy," Applied Mathematics and Computation, vol. 253, pp. 193-204, 2015.

[16] X. Xin and Y. Liu, "Reduced-order stable controllers for twolink underactuated planar robots," Automatica, vol. 49, no. 7, pp. 2176-2183, 2013.

[17] A. Zhang, J. She, X. Lai, and M. Wu, "Motion planning and tracking control for an acrobot based on a rewinding approach," Automatica, vol. 49, no. 1, pp. 278-284, 2013.

[18] X. Xin, S. Tanaka, J. She, and T. Yamasaki, "New analytical results of energy-based swing-up control for the Pendubot," International Journal of Non-Linear Mechanics, vol. 52, pp. 110-118, 2013.

[19] M. W. Spong, "Swing up control of the acrobot using partial feedback linearization," IFAC Proceedings Volumes, vol. 27, no. 14 , pp. 833-838, 1994.

[20] M. Spong, "The swing up control problem for the acrobot," IEEE Control Systems Magazine, vol. 15, no. 1, pp. 44-55, 1995.

[21] X. Lai, Z. Cai, J.-H. She, and Y. Ohyama, "Fuzzy control strategy for acrobots combining model-free and model-based control," IEE Proceedings-Control Theory and Applications, vol. 146, no. 6, pp. 505-510, 1999.

[22] I. Fantoni, R. Lozano, and M. W. Spong, "Energy based control of the pendubot," IEEE Transactions on Automatic Control, vol. 45, no. 4, pp. 725-729, 2000.

[23] X. Xin and M. Kaneda, "Analysis of the energy-based swingup control of the Acrobot," International Journal of Robust and Nonlinear Control, vol. 17, no. 16, pp. 1503-1524, 2007.

[24] A. Zhang, J. She, X. Lai, and M. Wu, "Global stabilization control of Acrobot based on equivalent-input-disturbance approach," in Proceedings of the 18th IFAC World Congress, pp. 14596-14601, Milano, Italy, August 2011.

[25] F. Bullo, A. Lewis, and K. Lynch, "Controllable kinematic reductions for mechanical systems: concepts, computational tools, and examples," in Proceedings of the International Symposium on Mathematical Theory of Networks and Systems, pp. 1-16, Leuven, Belgium, 2002.

[26] C. Popescu, Y. Wang, and Z. Roth, "Passivity based control of spring coupled underactuated horizontal double pendulum," in Proceedings of the Florida Conference of Recent Advances in Robotics, pp. 1-17, Florida, USA, May 2003.

[27] H. Khalil, Nonlinear Systems, Prentice-Hall, New Jersey, USA, 3rd edition, 2002. 